\title{
Analysis of Electrocardiogram Among 108 Patients with Brucella
}

\section{Li-Hong Lu \\ Yu-Qing Song \\ Qi-Ming Wu \\ Zhao Wang \\ Yong-Fu Chen \\ Qian Dong \\ Xiao-Tao Han}

Department of Cardiology, Beijing Ditan Hospital, Capital Medical University, Beijing, I000I5, People's Republic of China
Correspondence: Qi-Ming Wu

Department of Cardiology, Beijing Ditan Hospital, Capital Medical University, No. 8 of Jingshun Street, Chaoyang District, Beijing, 1000I5, People's Republic of China

Tel +86 I38 01009058

Fax +86 10-84322988

Email lulihong1954@I63.com
Objective: To improve our knowledge of cardiac manifestations caused by brucellosis through analyzing abnormal electrocardiograms of patients infected with brucella.

Methods: A total of 108 cases were enrolled, and their electrocardiograms were analyzed and summarized retrospectively.

Results: Among 108 cases, 77 (71.3\%) had a normal electrocardiogram, and 31 (28.7\%) had an abnormal electrocardiogram. There were 13 cases with nodal tachycardia (12\%), 9 cases with sinus bradycardia (8\%), 7 cases with sinus arrhythmia (6\%), 8 cases with left ventricular high voltage (7\%), 13 cases with abnormal ST segment and T wave (12\%), 2 cases with abnormal Q wave (1.85\%), 3 cases with complete right bundle branch block (2.78\%), 3 cases with ventricular premature beat $(2.78 \%), 1$ case with left anterior fascicular block $(0.9 \%), 1$ case with first degree a-v block $(0.9 \%), 1$ case with QT internal prolongation $(0.9 \%), 1$ case with poor $\mathrm{R}$ wave progression $(0.9 \%)$, and 1 case with short PR interval $(0.9 \%)$.

Conclusion: The cardiac manifestations of brucellosis were rare, but the mortality was high. Patients with abnormal electrocardiogram should have improved echocardiography in time. Early detection of the abnormal electrocardiogram could give a hint of cardiac damage to avoid the serious consequences.

Keywords: brucella, brucellosis, ECG, manifestations, cardiac manifestations

\section{Introduction}

Brucellosis is an anthropozoonosis caused by brucella. In recent years, the incidence rate of brucellosis in the world gradually increased year by year. ${ }^{1,2}$ Brucellosis, a systemic disease involving multiple organs and systems, is characterized by long-term recurrent fever, hyperhidrosis, feebleness, arthralgia, hepatomegaly and splenomegaly. Endocarditis caused by brucella was a rare disease and reported little abroad and at home recently. ${ }^{4-9}$ Brucellosis can invade multiple organs and systems, which usually happened in the bone and joint, and involved urogenital system, respiratory system, digestive system, nervous system, blood system, skin and eyes. ${ }^{10,11}$ The involvement of heart was rare, and characterized by endocarditis, myocarditis, pericarditis and endarteritis, $^{12}$ and endocarditis that happened in the $1-2 \%$ patients affected was the main cause of death from brucellosis. Recently, the incidence rate of the infective endocarditis was only $0.4 \%$, which was usually characterized by fever, cardiac murmur, hepatomegaly, and splenomegaly. Between 3-11 months after the appearance of clinical symptoms of brucella infection, neoplasm in the heart valve and heart failure were found.

Although damage of cardiovascular system caused by brucellosis was relatively rare, once the heart or central nervous system was invaded by brucella, patients' lives 
were in danger, and this was the main cause of death from the disease. ${ }^{3}$ When invading the heart, brucella could give rise to endocarditis, myocarditis, pericarditis, etc., on which the reports were few, let alone electrocardiogram results. Electrocardiograms are always used as early warning of cardiac damage. In our study, the electrocardiograms of 108 cases in our hospital from Sep 2012 to Jun 2018 were analyzed retrospectively to explore the possible cardiac abnormalities in the hope of early detection and treatment.

\section{Materials and Methods}

\section{The Source of Materials}

This was a retrospective study. 108 cases were diagnosed as brucellosis in the Beijing Ditan Hospital Capital Medical University from Sep 2012 to Jun 2018. Their data of manifestations and electrocardiograms was collected. The selection of ECG changes of interest is based on previous studies. ${ }^{3,4}$ The ECG was performed on the first day of admission. The Ethics approval was obtained from the hospital's institutional review board, and informed consent was obtained from every patient.

\section{Diagnostic Criteria}

Standards of diagnosis and stages were from the guidelines of diagnosis and treatment of brucellosis issued by the Ministry of Health in 2012.

\section{Statistical Analysis}

Data were analyzed using SPSS version 22.0. The measurement data were expressed by mean \pm standard deviation. The enumeration data were expressed by case, and the percentage was calculated.

\section{Results}

\section{The Basic Characteristics of Participants}

108 participants were included in this study. Their average age was $49.66 \pm 15.74$ years old. Among them, there were 83 men with an average age of $49.47 \pm 16.33$ years old, and 25 women with an average age of $50.28 \pm 13.27$ years old. There were 32 patients with abnormal sinus rhythm prior to brucella infection.

\section{The Manifestations of 108 Cases}

There were 4 cases with spondylitis (3.7\%), 4 cases with arthritis (3.7\%), 2 cases with abnormal liver functions $(1.85 \%), 1$ case with meningitis $(0.9 \%), 1$ case with brain abscess $(0.9 \%), 1$ case with paravertebral abscess
Table I Manifestations of 108 Patients

\begin{tabular}{|l|l|l|}
\hline Complications & N/108 & Percentage \% \\
\hline Spondylitis & $4 / 108$ & $3.7 \%$ \\
Arthritis & $4 / 108$ & $3.7 \%$ \\
Paravertebral abscess & $1 / 108$ & $0.9 \%$ \\
Meningitis & $1 / 108$ & $0.9 \%$ \\
Brain abscess & $1 / 108$ & $0.9 \%$ \\
Chronic hepatitis & $1 / 108$ & $0.9 \%$ \\
Abnormal liver function & $2 / 108$ & $1.85 \%$ \\
\hline
\end{tabular}

$(0.9 \%), 1$ case with orchitis $(0.9 \%)$, and 1 case with chronic hepatitis $(0.9 \%)$. The details are shown in Table 1.

\section{The Electrocardiograms of 108 Cases}

Among the 108 cases, 77 (71.3\%) had a normal electrocardiogram, and 31 (28.7\%) had an abnormal electrocardiogram. There were 13 cases with nodal tachycardia (12\%), 9 cases with sinus bradycardia (8\%), 7 cases with sinus arrhythmia (6\%), 8 cases with left ventricular high voltage (7\%), 13 cases with abnormal ST segment and $\mathrm{T}$ wave (12\%), 2 cases with abnormal Q wave (1.85\%), 3 cases with complete right bundle branch block $(2.78 \%)$, 3 cases with ventricular premature beat $(2.78 \%), 1$ case with left anterior fascicular block $(0.9 \%), 1$ case with first degree a-v block $(0.9 \%), 1$ case with QT internal prolongation $(0.9 \%), 1$ case with poor $\mathrm{R}$ wave progression $(0.9 \%)$, and 1 case with short PR interval $(0.9 \%)$. The details are shown in Table 2.

\section{Discussion}

In the present study, due to the rare occurrence of brucellosis with cardiovascular disease, none of the 108 cases was found with cardiovascular disease, but there were 31 cases with an abnormal electrocardiogram (28.7\%), which might be caused by the inadequate attention paid to the cardiac damage. If the patients with an abnormal electrocardiogram had undergone further examination of their hearts, some disease related with heart might have been discovered. As manifestations related with heart could put patients' lives in danger, patients with brucellosis and abnormal electrocardiograms should have further examination to detect whether cardiac disease exists or not

Endocarditis caused by brucella is a rare disease and has been reported little abroad and at home recently. ${ }^{4-9} \mathrm{Ma}$ et al. ${ }^{13}$ reported 4 relevant cases characterized by recurrent fever and I-II grade of cardiac function. For the 4 cases, they did not have an increase of leucocyte count and 
Table 2 Types of Electrocardiogram

\begin{tabular}{|l|l|l|}
\hline Types & N/I08 & Percentage \% \\
\hline Normal electrocardiogram & $77 / 108$ & $71.3 \%$ \\
Abnormal electrocardiogram & $3 / / 108$ & $28.7 \%$ \\
Nodal tachycardia & $13 / 108$ & $12 \%$ \\
Sinus bradycardia & $9 / 108$ & $8 \%$ \\
Sinus arrhythmia & $7 / 108$ & $6 \%$ \\
Left ventricular high voltage & $8 / 108$ & $7 \%$ \\
Abnormal ST segment and Twave & $13 / 108$ & $12 \%$ \\
Abnormal Q wave & $2 / 108$ & $1.85 \%$ \\
Complete right bundle branch block & $3 / 108$ & $2.78 \%$ \\
Ventricular premature beat & $3 / 108$ & $2.78 \%$ \\
Left anterior fascicular block & $1 / 108$ & $0.9 \%$ \\
First degree a-v block & $1 / 108$ & $0.9 \%$ \\
QT internal prolongation & $1 / 108$ & $0.9 \%$ \\
Short PR interval & $1 / 108$ & $0.9 \%$ \\
QT internal prolongation & $1 / 108$ & $0.9 \%$ \\
Poor R wave increment & $1 / 108$ & $0.9 \%$ \\
\hline
\end{tabular}

category. For the patients with long disease course, they experienced anemia, and even a decrease of white blood cells happened in the most seriously ill patients. Their serum agglutination test was positive, and when blood was collected under fever and cultured, the positive rate was higher. $\mathrm{Ma}$ and $\mathrm{Li}$ reported that the brucellosis involved endocardium, which was characterized by fever, cardiac murmur, anemia and organ embolism caused by bacterial embolus falling off. ${ }^{14}$ Liu et al. reported that there were 60 of 229 cases with brucellosis experiencing the manifestations, and one of them suffered myocarditis $(0.44 \%)^{15}$

Besides, there were 31 cases with an abnormal electrocardiogram (28.7\%), and among them, patients with nodal tachycardia were the most, which might be related with the fever caused by infection. Sinus bradycardia, changes of ST segment and $\mathrm{T}$ wave, abnormal $\mathrm{Q}$ wave, premature ventricular beats, bundle branch block, poor $\mathrm{R}$ wave progression, etc. might be related with myocardial damage caused by infection. The other abnormal electrocardiogram might be caused by the changes of basis of electrocardiogram.

There are some limitations in our study. Firstly, this study was not a randomized controlled trial. Secondly, the sample size was limited, e.g., many findings were reflecting on $1 / 108(0.9 \%)$, thus another trial with large sample size was needed. Also, there was not enough information about clinical follow-up data, which should be further researched. Thirdly, there were not enough data about the repetitive ECG, which should be further researched. Fourthly, this study does not include structural changes, but only ECG findings. This is just a basic descriptive statistical analysis, no contingency analysis was performed. While very basic demographic data varied significantly in some variables (e.g. 3/4 of the study population were males), no statistical analysis was performed to examine outcomes in the context of an interesting finding (significant gender variation in the cohort).

In summary, brucella can involve multiple organs and systems in the whole body, with various clinical manifestations. Cardiovascular system involvement was relatively rare, which can be manifested as endocarditis, vasculitis and myocarditis. Endocarditis is an uncommon but leading cause of death. Electrocardiogram examination is simple and feasible. For patients infected with brucella who have an abnormal electrocardiogram, relevant examinations should be conducted in time to further clarify the heart condition.

\section{Ethics Approval and Consent to Participate}

This study was conducted in accordance with the Declaration of Helsinki. This study was conducted with approval from the Ethics Committee of Beijing Ditan Hospital, Capital Medical University. A written informed consent was obtained from all participants.

\section{Acknowledgments}

No funding or sponsorship was received for this study or publication of this article.

\section{Disclosure}

The authors report no conflicts of interest in this work.

\section{References}

1. Akhvlediani T, Clark DV, Chubabria G, et al. The changing pattern of human brucellosis: clinical manifestations, epidemiology, and treatment outcomes over three decades in Georgia. BMC Infect Dis. 2010;10(1):346. doi:10.1186/1471-2334-10-346

2. AI Dahouk S, Neubauer H, Henscl A, et al. Changing epidemiology of human brucellosis, Germany, 1962-2005. Emerg Infect Dis. 2007;13 (12):1895-1900. doi:10.3201/eid1312.070527

3. Reguera JM, Alarcrn A, MirMles F, et al. Brucella endocarditis: clinical, diagnostic, and therapeutic approach. Eur J Clin Microbiol Infect Dis. 2003;22(11):647-650. doi:10.1007/s10096-003-1026-z

4. Manias V, Nagel A, Mollerach A, et al. Brucella canis endocarditis: first documented ease in Argentina. Rev Argent Microbiol. 2013;45 (1):50-53.

5. Raju IT, Solanki R, Patnaik AN, et al. Brucella endocarditis-a series of five case reports. Indian Heart J. 2013;65(1):72-77. doi:10.1016/j. ihj.2012.12.017 
6. Yazıcı HU, Mert KU, Senol U, et al. A case with tricuspid valve brucella endocarditis presenting with acute right heart failure. Turk Kardiyol Dern Ars. 2012;40(4):364-367. doi:10.5543/tkda.2012.32067

7. Colomba C, Siracusa L, Rubino R, et al. A case of Brucella endocarditis in association with subclavian artery thrombosis. Case Rep Infect Dis. 2012;2012:581489.

8. Köse Ş, Serin Senger S, Ersan G, et al. Presentation of two cases diagnosed with Brucella endocarditis. Intern Med. 2012;51 (8):953-955. doi:10.2169/internalmedicine.51.7103

9. Mehanic S, Mulabdic V, Baljic R, et al. Brucella endocarditis in prosthetic valves. Mater Sociomed. 2012;24(Suppl 1):11-12. doi:10.5455/msm.2012.24.s11-s12

10. Hasanjani Roushan MR, Mohrez M, Smailnejad Gangi SM, et al. Epidemiological features and clinical manifestations in 469 adult patients with brucellosis in Babol, Northern Iran. Epidemiol Infect. 2004;132(6):1109-1114. doi:10.1017/S0950268804002833
11. Mantur BG, Amarnath SK, Shinde RS. Review of clinical and laboratory features of human brucellosis. Indian J Med Microbiol. 2007;25(3):188-202. doi:10.1016/S0255-0857(21)02105-8

12. Herrick JA, Lederman RJ, Sullivan B, et al. Brucella arteritis: clinical manifestations, treatment, and prognosis. Lancet Infect Dis. 2014;14 (6):520-526. doi:10.1016/S1473-3099(13)70270-6

13. Ma SF, Li XF, Shan XF. Brucella infective endocarditis in 4 cases. Chin J Thorac Cardiovasc Surg. 2011;27(3):184.

14. Ma XZ, Li XY. Report of two cases of Brucella endocarditis. Chin J Infec Chemother. 2017;17(4):451-453.

15. Liu J, Fu CT, Chen XH. Clinical analysis of 229 cases of brucellosis. J Zhejiang Univ. 2012;41(6):677-680.

\section{Publish your work in this journal}

The International Journal of General Medicine is an international, peer-reviewed open-access journal that focuses on general and internal medicine, pathogenesis, epidemiology, diagnosis, monitoring and treatment protocols. The journal is characterized by the rapid reporting of reviews, original research and clinical studies across all disease areas. The manuscript management system is completely online and includes a very quick and fair peer-review system, which is all easy to use. Visit http://www.dovepress.com/ testimonials.php to read real quotes from published authors. 